\title{
Practical considerations on optimising multistage decimation and interpolation processes
}

\author{
Xiangyu Zhu ${ }^{1}$, Yonghao Wang ${ }^{1}$, Wei Hu ${ }^{2,3}$, Joshua D. Reiss ${ }^{4}$ \\ ${ }^{1}$ DMT Lab, Birmingham City University, UK \\ ${ }^{2}$ College of Computer Science and Technology, Wuhan University of Science and Technology, China \\ ${ }^{3}$ Hubei Province Key Laboratory of Intelligent Information Processing and Real-time Industrial System, China \\ ${ }^{4}$ Centre for Digital Music, Queen Mary University of London, UK \\ xiangyu.zhu@mail.bcu.ac.uk, ${ }^{*}$ yonghao.wang@bcu.ac.uk, huwei@wust.edu.cn, joshua.reiss@qmul.ac.uk
}

\begin{abstract}
Multistage filter design is a complex multidimensional optimisation problem. The formulae for optimal design generally yield non-integer real numbers for the sample-rate-changing factors of multiple stages. Approaches yielding useful integer results have high computational cost and do not consider important multistage filter design properties. We have developed a simplified algorithm for directly searching the optimal integer results. Considering the most useful practical design parameters, optimal results can be approximated with a limited number of sets for any designs satisfying certain constraints, with negligible costs. This vastly simplifies the complexity of the problem.
\end{abstract}

Keywords: Multistage, filter design, FIR, decimation, interpolation.

\section{INTRODUCTION}

Oversampling and sigma-delta based converters have become popular in digital audio applications due to their high resolution and low hardware cost [1] [2] [3]. Most modern digital audio systems include some sample rate conversion processes in either software format or integrated circuits. The key components of sampling rate alteration are the decimation or interpolation filters, which alter the sampling rate and suppress unwanted digital aliasing and imaging, respectively.

The multistage filter structure can be up to 10 times more efficient than a single stage structure. Reference [4] [5] presented the theory and quantification of cost optimisation of multistage structures. Reference [6] [7] found that optimal solutions can be derived analytically by taking the partial differential equation (PDE) of the cost function, hence reducing it to a one dimensional problem without needing complex numerical search algorithms. However optimal solutions are often groups of non-integer real numbers that cannot be implemented in practical systems. Manual adjustment of results is needed, one still needs to retreat to numerical methods to solve the equations, and for each design, the roots of the equation must be put back into a cost function to find the optimal solution set. Alternatively, one can yield the integer solution directly. Reference [8] represents this problem in the integer domain using set theory, and then performs integer factorization. Reference [9] showed that the problem can be solved using exhaustive search or a genetic algorithm.

We show that properties of solutions allow simplification of the search algorithm (Section II). Based on distribution of the solution sets, we propose a new search algorithm and use it to generate optimal solution lookup tables for practical designs
(Section III). A balanced trade-off strategy is developed to find a best solution set for both computational and memory area cost (Section IV). Conclusions are given in the last section.

\section{DESCRIPTION OF THE BACKGROUND THEORY}

The digital FIR filter design with very narrow transition band, high attenuation stopband and flat passband results in large order filters. The digital anti-aliasing and anti-image filters used in oversampling based high resolution ADC/DAC belong to this category [10] [11]. The classical approach is to use multistage design so that the design specification of each individual filter (stage) can be relaxed. Given the overall sampling rate changing factor $D$, each stage in a $K$ stage filter alters the sampling rate by $D_{i}$ where $D=\prod_{i=1}^{K} D_{i}$. For a $K$ stage filter and given design specifications, the problem is to find the set $\left\{D_{i}\right\}$ such that $D$ is optimal in terms of computation or memory area cost.

Let $\delta_{p}$ and $\delta_{s}$ represent the tolerance in the magnitude response in the passband and stopband respectively. $\Delta f=$ $\frac{f_{s}-f_{p}}{f_{s}}$, where $f_{s}$ is the stopband edge and $f_{p}$ is the passband edge, and $f_{r 0}$ is the input signal's sampling frequency. The computational cost $R_{T}$ is the total number of multiplies and adds per second $(M A D S / s)$, and given by (1) [4] [5].

$$
R_{T}=D_{\infty}\left(\frac{\delta_{p}}{K}, \delta_{s}\right) f_{r 0} S
$$

Where

$$
\begin{aligned}
& D_{\infty}\left(\delta_{p}, \delta_{s}\right)=[ 5.309 \times 10^{-3}\left(\log _{10} \delta_{p}\right)^{2}+7.114 \\
&\left.\times 10^{-2}\left(\log _{10} \delta_{p}\right)-0.4761\right] \log _{10} \delta_{s} \\
&-\left[2.66 \times 10^{-3}\left(\log _{10} \delta_{p}\right)^{2}\right. \\
&\left.+0.5941\left(\log _{10} \delta_{p}\right)+0.4278\right] \\
& S=\frac{2}{\Delta f} \frac{1}{\prod_{j=1}^{K-1} D_{j}}+\sum_{i=1}^{K-1} \frac{D_{i}}{\left(\prod_{j=1}^{i} D_{j}\right)\left(1-\left(\frac{2-\Delta f}{2 D}\right) \prod_{j=1}^{i} D_{j}\right)}
\end{aligned}
$$

The total memory storage cost of such a filter is given by

$$
N_{T}=D_{\infty}\left(\frac{\delta_{p}}{K}, \delta_{s}\right) G T
$$


Where $G$ is a proportionality constant that relates to the implementation of filter coefficients and $T$ is given by

$$
T=\frac{2}{\Delta f} \frac{D}{\prod_{j=1}^{K-1} D_{j}}+\sum_{i=1}^{K-1} \frac{D_{i}}{1-\alpha \prod_{j=1}^{i} D_{j}}
$$

where $\alpha=\frac{2-\Delta f}{2 D}$.

To minimise $R_{T}$ is to minimise $S$ in (3) and to minimise $N_{T}$ is to minimise $T$ in (5). $S$ and $T$ are only dependent on the $D_{i}$ and $\Delta f$.

Reference [6] and [7] took a PDE approach to cost functions (3) and (5), treating $D_{i}$ as continues real value. For example, finding $D_{1}$ in a 3 -stage design can be formulated as a roots finding problem:

$$
\frac{\partial T}{\partial D_{1}}=-\frac{2 D}{\Delta f D_{1}^{2} D_{2}}+\frac{1}{\left(1-\alpha D_{1}\right)^{2}}+\alpha \sqrt{\frac{2 D}{\Delta f} \frac{1}{D_{1}}} D_{2}=0
$$

Often solving (6) requires numeric methods and results in complex and irrational roots.

Reference [8] and [9] directly search integer sets of $\left\{D_{i}\right\}$ that are factors of $D$, using exhaustive search or a genetic algorithm to produce integer valued optimal results, but did not taking the properties of the real valued solution into account.

In this work, we observe the distributions of both real valued and integer valued optimal solution sets. We find both of these follow certain regular patterns. This enables us to vastly simplify the optimisation problem and the size of the problem. The findings are discussed in the following section.

\section{KNOWLEDGE-BASED SEARCH AND LOOKUP TABLES}

Observing from the experiments' results of both real-valued and integer valued optimal solutions, there are three important properties of the distributions of optimal solutions for both optimal computational cost and memory storage cost:

a) $\left\{D_{i}\right\}$ is always in descending order for multistage decimation and in ascending order for multistage interpolation.

The larger value of $D_{i}$ means the larger decimation or interpolation factors stage $i$. In order to minimise the narrow transition band effects on high sampling frequency (oversampled), it is understandable to have larger $D_{i}$ close to the higher sampling frequency end.

The experiments show that the average real valued solution of smallest value of $D_{i}$ for 3-stage design is around $2.65\left(D_{3}\right)$ and for 4-stage design is around $1.52\left(D_{4}\right)$ for decimation process. These number is close to integer number 2 that is the minimum sampling rate changing factor, and is close to the lowest sampling frequency stage.

b) $\Delta f$ is related to the width of transition band. The variation of $\Delta f$ changes the order of the filter but not the sampling rate changing factor of each stage.

This is because for same overall value $D$, the distribution of $D_{i}$ still follows the trend of property a) regardless of filter order. According to [6] [7], $\Delta f$ has only a small effect on the results. Also because the results are not integer, rounding is needed and often, the difference due to using different $\Delta f$ is smaller than the difference caused by the rounding process.

c) Because of a), we need to test the optimal set by cost functions only for highly composite number (non-prime) $D$ that can be factorised more than number of stages $K$.

Thus, search for integer valued solutions can be informed by a), and because of b) and c), the problem size is considerably small than it appeared to be.

Fig. 1 shows 3D plots of the 3 stage real-valued optimal solution distribution for optimising $R_{T}$ (Fig.1.a) and $N_{T}$ (Fig. 1.b). The real valued optimal result sets $\left\{D_{1}, D_{2}, D_{3}\right\}$ are formed from three independent disjoint surfaces with different $D<5000$ and $0<\Delta f<0.5$. The values of lowest surface is close to the minimum value of interpolation and decimation factor 2 . The optimal integer valued solutions follow a similar trend. Fig 2 demonstrates this for $D=2^{n}$.

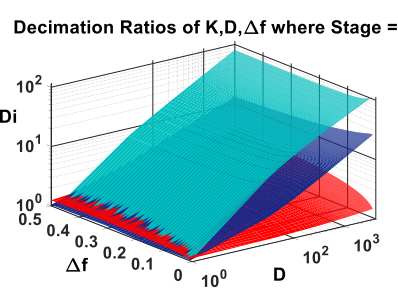

(a)

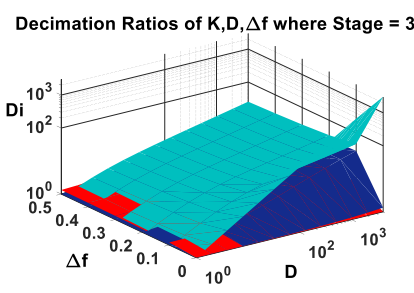

(b)
Figure 1 Real-valued solution sets distribution

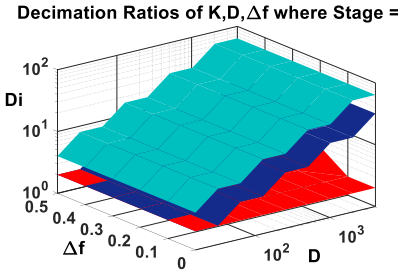

(a)

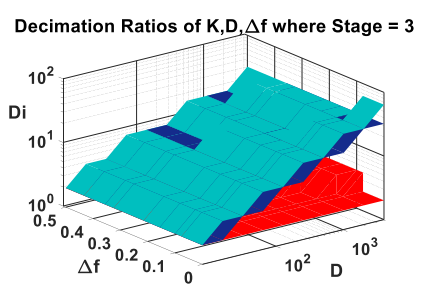

(b)
Figure 2 Integer solution sets distribution for $D=2^{n}$

The simplified search algorithm is described in Table I:

TABLE I PSEUDOCODE OF OPTIMAL INTEGER VALUED SOLUTION SEARCH ALGORITHM

For $D \in \mathbb{N}_{1}$ and represented as a prime factorization:

$$
D=p_{1}^{d_{1}} * p_{2}^{d_{2}} * p_{3}^{d_{3}} \ldots * p_{r}^{d_{r}}
$$

where $p_{i}$ is a prime number and $d_{i}$ the corresponding exponent,

1) Check whether $D$ can be factorized into $M$ unique sets of $k$ (stage) factors

$$
M=\left\{\begin{array}{c}
\left\{D_{1.1}, D_{2.1}, \ldots D_{k .1}\right\} \\
\left\{D_{1.2}, D_{2.2}, \ldots D_{k .2}\right\} \\
\vdots \\
\left\{D_{1 . m}, D_{7 . m}, \ldots D_{k . m}\right\}
\end{array}\right\}
$$

2) Sort $M$ so that $\left\{D_{i .1}>D_{i .2}>\cdots>D_{i . k}\right\}$

3) Substitute sets into (3) or (5) and find the set with the minimum solution.

The algorithm simplifies the search since we only care about unique sets (e.g., $\{8,4,2\}$ is equivalent to $\{4,8,2\}$ ), and 
we sort candidate sets as descending ordered sequences. Only when the number of $D$ 's factors is larger than the required number of stages, the step 2) sorting required. For example, for $D<5000,3$ stage decomposition, only 1692 (33.8\%) numbers can be factorised in different unique sets of 3 factors that need to be put back into the cost function. For typical design value $\Delta f=0.18$ and $D<5000$ and 2, 3, 4 stage design, our method provides $85.4 \%$ average reduction when compared with exhaustive search in terms of the number of cost function tests, and $65 \%$ computing time reduction. Computing time was averaged over 100 iterations using a standard Intel Core i7 based PC.

In addition, the variation of $\Delta f$ does not cause much change in the optimal integer values. For the same example of $D<5000$ and 3 stage case, within the 1692 cases that have possible multiple solutions, in 994 (59\%) cases for computational cost optimisation and $1167(69 \%)$ cases for memory cost optimisation, the optimal solution sets change only once or twice over the (0 to 0.5$) \Delta f$ region.

Table II summarises 15 popular ADC/DAC chipsets used in audio devices from on-board sound cards to professional mixers. The supported sampling frequencies $F_{S}$ range from 44.1 $\mathrm{kHz}, 48 \mathrm{kHz}, 96 \mathrm{kHz}$ with different supported filter type configurations such as low latency, sharp or slow roll off, etc. $\Delta f$ is within $0.08-0.44$ with most common designs between 0.15 and 0.4

TABLE II SUMMARY OF $\triangle f$ VALUES OF COMMON OVERSAMPLING BASED AUDIO ADC/DAC DESIGN SPECIFICATIONS

\begin{tabular}{|c|c|c|c|}
\hline Type & $\operatorname{Max} \boldsymbol{f}_{p}$ & $\operatorname{Min} f_{s}$ & $\operatorname{Min} \Delta f$ \\
\hline AD1871 & $0.45 F_{S}$ & $0.55 F_{S}$ & 0.17 \\
\hline AD7768 & $0.43 F_{S}$ & $0.50 F_{S}$ & 0.14 \\
\hline AD1974 & $0.44 F_{S}$ & $0.56 F_{S}$ & 0.21 \\
\hline ADAU1966A & $0.36-0.45 F_{S}$ & $0.55-0.64 F_{S}$ & $0.18-0.44$ \\
\hline PCM1807 & $0.45 F_{S}$ & $0.58 F_{S}$ & 0.22 \\
\hline PCM4220 & $0.42-0.45 F_{S}$ & $0.55-0.58 F_{S}$ & $0.18-0.28$ \\
\hline PCM1794A & $0.46-0.49 F_{S}$ & $0.55-0.73 F_{S}$ & $0.11-0.37$ \\
\hline PCM5242 & $0.40-0.47 F_{S}$ & $0.55 F_{S}$ & $0.15-0.18$ \\
\hline CS5364/66/68 & $0.45-0.47 F_{S}$ & $0.58-0.68 F_{S}$ & $0.19-0.34$ \\
\hline CS5381 & $0.45-0.47 F_{S}$ & $0.58-0.68 F_{S}$ & $0.19-0.34$ \\
\hline CS4398 & $0.499 F_{S}$ & $0.55-0.58 F_{S}$ & $0.09-0.14$ \\
\hline WM8740 & $0.27-0.45 F_{S}$ & $0.46-0.49 F_{S}$ & $0.08-0.41$ \\
\hline WM8741 & $0.4 F_{S}$ & $0.5 F_{S}$ & 0.2 \\
\hline ALC885 & $0.45 F_{S}$ & $0.60 F_{S}$ & 0.25 \\
\hline CS4207 & $0.45-0.499 F_{S}$ & $0.55-0.60 F_{S}$ & $0.09-0.25$ \\
\hline
\end{tabular}

We use a bisection method to find the $\Delta f$ points where optimal solutions change. For common 2, 3, 4 stage filter design, two groups of lookup tables for computational and area costs can be generated from the algorithm. They are small since optimal solutions are smooth over useable design specification ranges.

Fig. 3 shows changing of optimal solution sets $\left\{D_{i}\right\}$ against $D$ and $\Delta f$. The $Z$ axis value is calculated from (7), where $\omega$ is a weighting factor, and $\sigma\left(D_{i}, K\right)$ is the standard deviation of optimal set $\left\{D_{i}\right\}$. It provides information regarding both $\prod D_{i}$ and the distribution value of $D_{i}$ within a solution set. Since the elements of set $\left\{D_{i}\right\}$ are descending or ascending,
$\sigma\left(D_{i}, K\right)$ indicates the slope of the value changing across stages. The same value of $\mathrm{Z}$ indicates same set of $\left\{D_{i}\right\}$ being chosen.

$$
Z=\omega D+\sigma\left(D_{i}, K\right)
$$

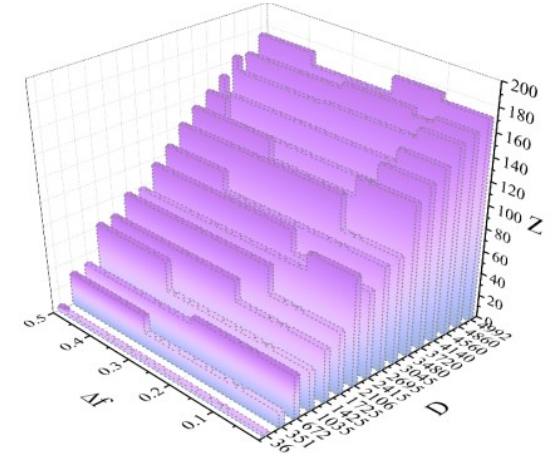

Figure 3 Changing of optimal value against $\Delta f$ for some highly composite number $D$

Fig. 3 shows that optimal integer solution sets can be the same values for a large range of design specification $\Delta f$. Similar figures can be produced for both optimal area cost and computational cost sets. Thus, we can create lookup tables to store these optimal solutions with the critical values of $\Delta f$ that cause changes in optimal solution sets. The structure of a database of such tables is depicted in Fig. 4. Using our algorithm to generate $K=2,3,4$ stage design tables for both memory and computational cost, with $1<D<5000$ and $0<\Delta f<0.5$, there are 15,783 total optimal computational cost sets and 17,622 total optimal memory usage sets in the three tables.

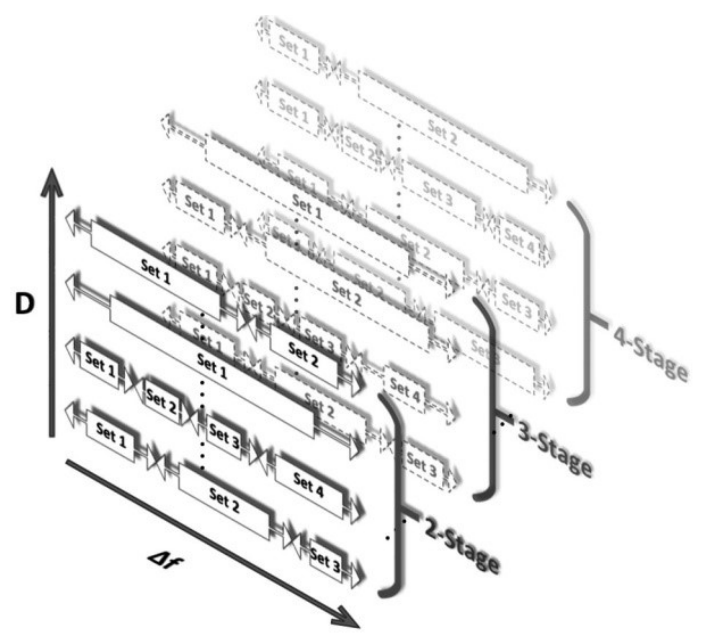

Figure 4 depiction of optimal solution sets lookup tables of computational cost or memory storage cost

\section{TRADEOFF STRATEGY FOR MINIMISATION OF BOTH AREA AND COMPUTATIONAL COST}

In practise, only one solution can be used in a system. A trade-off strategy based on error effects was developed to find a best solution set for both computational and memory area cost within the lookup tables.

$$
\begin{aligned}
& \text { when }\left\{D_{i}\right\}_{m} \neq\left\{D_{i}\right\}_{c} \\
& \text { if } \frac{C_{m}-C_{c}}{C_{c}}>\frac{M_{c}-M_{m}}{M_{m}} \text { choose }\left\{D_{i}\right\}_{c} \\
& \text { else choose }\left\{D_{i}\right\}_{m}
\end{aligned}
$$


Where:

$\left\{D_{i}\right\}_{c}$ is the integer valued solution set for optimal computational cost.

$\left\{D_{i}\right\}_{m}$ is the integer valued solution set for optimal memory usage cost. set.

$C_{c}$ is computational cost with optimal computational cost

$C_{m}$ is computational cost with optimal memory usage set.

$M_{C}$ is memory usage with optimal computational cost set.

$M_{m}$ is memory usage with optimal memory usage set.

To evaluate the error effects of this trade-off strategy, we evaluated the average error of computational cost: $S_{\text {diff }}=$ $\left(\sum_{i=1}^{N} \frac{C_{i}-C_{c i}}{C_{c i}}\right) / N$, and the average error of memory cost: $T_{\text {diff }}=\left(\sum_{i=1}^{N} \frac{M_{i}-M_{m i}}{M_{m i}}\right) / N$, where $N$ is total number of design cases tested; $C_{i}$ and $M_{i}$ are the actual computational cost and memory usage when the trade-off sets being used; $C_{c i}$ and $M_{m i}$ are the computational cost and memory usage when the corresponding optimal sets being used.

For 3 stage design with $0<\Delta f<0.5$ and $0<D<5000$, the average error is $0.26 \%$ for $S_{\text {diff }}$ and $8.88 \%$ for $T_{\text {diff }}$.

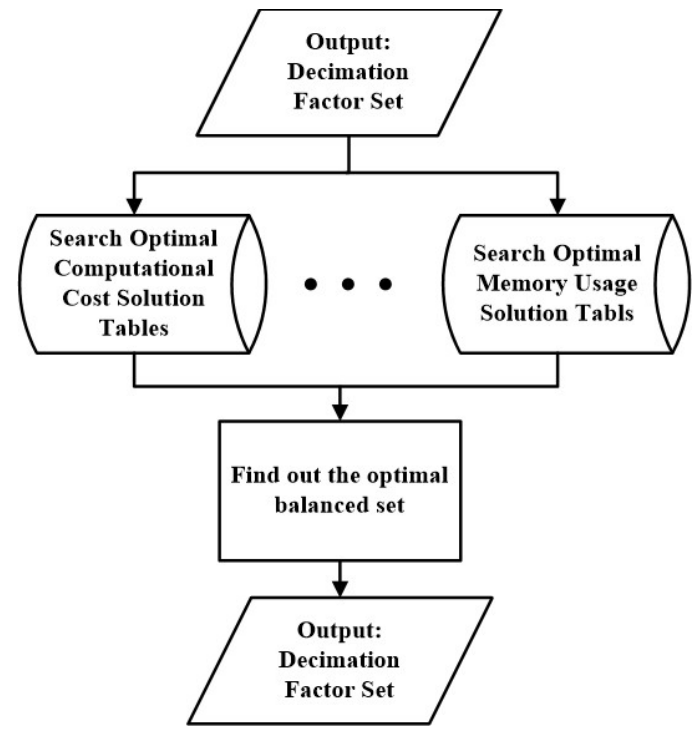

Figure 5 Follow chart of overall database query algorithm

Fig. 5 shows the flowchart for finding semi-optimal solutions for both constraints. It is worth mentioning that this method can be altered with extra weighting factor to consider actual implementation effects. The design parameter $\Delta f, D$ and different platform realisation techniques might have influence of actual selection decisions. Further work needs to be done to decide the form of weighting factor.

\section{CONCLUSION}

We provided new experimental results and analysis concerning optimal multistage multirate FIR filter design. The results show that the optimal solution set possesses important properties that allow for knowledge-based search, and that the number of optimal solution sets are limited since they do not change the set values over a large range of design specifications. This motivates the design of multiple lookup tables of solution sets. A trade-off strategy is employed to produce semi-optimal solution sets that reduce computational and memory usage costs.

Our approach was derived based on classical individual optimal FIR filter design [12] in terms of theoretical number of multiplies and adds needed, and the memory storage for storing signal samples and filter coefficients. There are a set of different filter computation reduction methods based on creating one-stage two-filters system where one filter $\mathrm{B}(\mathrm{z})$ shapes the passband and another filter $A(z)$ shapes the stopband. The passband filter $B(z)$ works at lower sampling rate of multirate system . It will be interesting to investigate how their work performs at very high sampling rate alteration $\mathrm{D}$ in comparison with this work [13] [14] [15]. Other research has looked into further bit-level optimisation of filter coefficients in a multistage architecture [16] [17], and specific application cases [18] [19]. It would be interesting to see how the optimal filter can be applied to those cases. The number of stages and their rate changing factors also affect filter latency [20], and computational cost, memory usage and their realisation methods affect the energy consumption. An interesting research direction would be to look into a model that considers these additional aspects of optimal multistage design.

\section{ACKNOWLEDGMENT}

The algorithms herein are available under MIT open source license from https://github.com/wyonghao/MultiStageDesign.

\section{REFERENCES}

[1] W. Kester, "A Brief History of Data Conversion: A Tale of Nozzles, Relays, Tubes, Transistors, and CMOS,"IEEE Solid-State Circuits Magazine, vol. 7, no. 3, pp. 16-37, Summer 2015.

[2] S. Norsworthy, et al. "Delta-sigma data converters: theory, design, and simulation" IEEE press New Jersey,. Ch13, p 406-446, 1996

[3] Kester, W. Mixed-Signal and DSP Design Techniques. Norwood, MA: Analog Devices, Ch. 3, pp. 16-17. 2000

[4] R. Crochiere and L. Rabiner, "Optimum FIR digital filter implementations for decimation, interpolation, and narrow-band filtering", IEEE Trans. Acoust. Speech Signal Process., v. 23, no. 5, pp. $444-456$, Oct. 1975.

[5] R. E. Crochiere and L. Rabiner, "Interpolation and decimation of digital signals - A tutorial review", Proc. IEEE, vol. 69, no. 3, pp. 300-331, 1981.

[6] M. W. Coffey, "Optimizing multistage decimation and interpolation processing", IEEE Signal Process. Lett., vol. 10, no. 4, pp. 107-110, 2003.

[7] M. W. Coffey, "Optimizing Multistage Decimation and Interpolation Processing mdash;Part II", IEEE Sig. Proc. Lett., 14 (1), pp. 24-26, 2007.

[8] Der-Feng Huang, "The direct integer factorization approach to the Crochiere and Rabiner multistage FIR designs for multirate systems", 3rd Int. Symp. Image Sig. Proc. Analysis (ISPA), v. 2, p1060-65, 2003.

[9] D.-F. Huang \& S.-R. Hung, "The optimum design of multistage multirate FIR filter for audio signal sampling rate conversion via a genetic algorithm approach",2nd Int. Cong. Image Sig. Proc. (CISP), 2009

[10] Lesso, P. and Magrath, A. J. "An Ultra High Performance DAC with Controlled Time-Domain Response" $119^{\text {th }}$ AES Conv. 119, Oct. 2005

[11] Craven, Peter G. "Antialias Filters and System Transient Response at High Sample Rates." Journal of the Audio Engineering Society 52.3, 2004

[12] T.W. Parks and J.H. McClellan, "Chebyshev approximation for nonrecursive digital filters with linear phase," IEEE Trans. Circuit Theory, vol. CT-19, no. 2, pp. 189-194, 1972. 
[13] T. Saramäki, "A New Class of Linear-Phase FIR Filters for Decimation, Interpolation, and Narrow-Band Filtering," Proc. IEEE Int. Symp. Circuits Syst., pp. 808-811, Rome, Italy, May 10-12, 1982

[14] T. Saramäki, "A class of linear-phase FIR filters for decimation, interpolation, and narrow-band filtering," IEEE Transactions on Acoustics, Speech, and Signal processing, vol. ASSP-32, no. 5, pp. 1023-1036, October 1984.

[15] P. Arian and T. Saramäki, "A systematic technique for optimizing onestage two-filter linear-phase FIR filters for sampling rate conversion," in Proc. 2003 IEEE International Symposium on Circuits and Systems, Bangkok, Thailand, May 25-28, 2003, vol. 4, pp. 197-200.

[16] A. Blad et al, "Bit-level optimized FIR filter architectures for high-speed decimation applications," IEEE Int. Symp. Circ. Syst., Seattle, 2008.
[17] A. Shahein, et al, "A Novel Hybrid Monotonic Local Search Algorithm for FIR Filter Coefficients Optimization," IEEE Trans. Circ. Syst. I: Regular Papers, 59 (3), p 616-627, March 2012.

[18] M. Mottaghi-Kashtiban, et al, "Optimum Structures for Sample Rate Conversion from $\mathrm{CD}$ to DAT and DAT to $\mathrm{CD}$ Using Multistage Interpolation and Decimation," IEEE Int. Symp. Sig. Proc. Inf. Tech., Vancouver, 2006

[19] S. Liu, W. Jiang, and M. Zhang. "Dual-channel multiplexing technology and its realization in interpolation filter in stereo audio sigma-delta DAC". Analog Integrated Circuits and Signal Processing, 81 (2), Nov. 2014

[20] Y. Wang, J. D. Reiss, "Time domain performance of decimation filter architectures for high resolution sigma delta analogue to digital conversion", 132nd AES Convention, Budapest, April 26-29, 2012 